\title{
Airway symptoms and lung function in pipelayers exposed to thermal degradation products from MDI-based polyurethane
}

\author{
K Jakobsson, K Kronholm-Diab, L Rylander, L Hagmar
}

\begin{abstract}
Objectives-To study the prevalence of symptoms from the eyes and the upper and lower respiratory tract, lung function, and immunological sensitisation towards isocyanates in pipelayers exposed to thermal degradation products from methylene diphenyl diisocyanate (MDI)-based polyurethane (PUR).

Material and methods-50 presently active and 113 formerly active pipelayers were examined. Also, 65 unexposed workers were investigated for comparison. The one year prevalence of symptoms and smoking history (questionnaire data), lung function (vital capacity (VC) and forced expiratory volume in one second $\left(F E V_{1}\right)$, and atopy (positive skin prick tests towards standard allergens) were assessed among pipelayers and controls. For the pipelayers, the presence of work related symptoms and estimates of isocyanate and welding exposure were obtained from an interview. Skin prick tests towards specific isocyanate antigens and determinations of IgE-MDI and IgG-MDI in serum were also performed.
\end{abstract}

Results-The prevalence of episodes (more than once a month) of irritative eye symptoms, congestion of the nose, and soreness or dryness in the throat was much higher among the PUR pipelayers than among the controls. Most of the pipelayers with symptoms reported that these had started and occurred in relation to the PUR welding tasks. Presently active pipelayers with recent high PUR exposure showed a significant reduction of $F E V_{1}$ compared with the controls. The estimated reduction, adjusted for smoking, was $-0.31(P=0.04)$. There was no confounding effect of ordinary welding. None of the pipelayers showed positive skin prick reactions against the specific isocyanate antigens used, or positive IgE-MDI, and only two had increased IgG-MDI.

Conclusions-The findings indicate that exposure to thermal degradation products from MDI-based polyurethane has adverse effects on the mucous membranes and airways.

(Occup Environ Med 1997;54:873-879)

Keywords: methylene diphenyl diisocyanate; respiratory disease; construction industry
Isocyanates are agents responsible for a substantial proportion of occupational asthma. ${ }^{1}$ In industrial handling, methylene diphenyl diisocyanate (MDI) has been considered to have a low potential in inducing respiratory disease compared with other isocyanates, because of its low vapour pressure. However, especially in connection with spraying or heating, MDI has been associated with asthmatic, as well as systemic reactions. ${ }^{2-5}$ The pathogenetic mechanisms are still controversial; irritant, immunological and pharmacological mechanisms have been suggested, and may well coexist. Moreover, irritative conjunctival and upper airway symptoms may also be prevalent after exposure to $\mathrm{MDI},{ }^{36}$ but have arroused less interest.

In the present study we examined pipelayers who had been welding district heating pipes insulated with polyurethane (PUR) foam. This foam is a complicated structure formed by polymerisation of a mixture of reagents mainly containing 4,4'-MDI, 2,2'-MDI, 2,4'-MDI, and polyethers with one or two hydroxyl groups. If the foam is not thoroughly removed from the mild steel pipe before welding, the pipelayers are exposed not only to the welding fumes but also to thermal degradation products from the foam. These products include numerous different isocyanates, aminoisocyanates and amine compounds. ${ }^{7}$ Workplace monitoring with a filtertape instrument has indicated considerable exposure ( $M$ Dalene, personal communication). A biomarker, 4,4-methylenedianiline, has been used to assess isocyanate exposure among these pipelayers. ${ }^{8}$ In the present study the aims were to investigate the prevalence of symptoms from the eyes and the upper and lower respiratory tract, and lung function. Further, immunological tests were performed to detect possible sensitisation.

\section{Material and methods}

PIPELAYERS: STUDY GROUP AND EXPOSURE Thirty one companies engaged in PUR pipelaying in the south of Sweden were identified from telephone directories and from suppliers of pipes. From all but one, we obtained lists of present and former employees who had ever participated for at least four weeks in such work. Information on the study was sent to each subject, together with a short enquiry on work related symptoms, duration of work with PUR, and smoking. The entry criteria were met by 228 men, of whom $163(71 \%)$ participated in the total examination; for the remain- 
Table 1 Age, smoking habits, and exposure among pipelayers and controls

\begin{tabular}{|c|c|c|c|}
\hline & \multicolumn{2}{|l|}{ PUR pipelayers } & \multirow[b]{2}{*}{ Controls } \\
\hline & Presently active & Formerly active & \\
\hline Subjects (n) & 50 & 113 & 65 \\
\hline $\mathrm{Age}^{\star}$ & $38(25,53)$ & $49(35,61)$ & $41(22,59)$ \\
\hline \multicolumn{4}{|l|}{ Smoking habits: } \\
\hline Lifelong non-smoker (n (\%)) & $18(36)$ & $25(22)$ & $34(52)$ \\
\hline Ex-smoker (n (\%)) & $19(38)$ & $59(52)$ & - \\
\hline Pack-years ${ }^{\star}$ & $14(2,27)$ & $14(2,33)$ & - \\
\hline Age at start of smoking ${ }^{\star}$ & $16(15,21)$ & $17(14,20)$ & - \\
\hline Current smoker (n (\%)) & $13(26)$ & $29(26)$ & $31(48)$ \\
\hline${\text { Pack-years }{ }^{\star}}$ & $12(1,33)$ & $21(4,40)$ & $14(5,37)$ \\
\hline Age at start of smoking ${ }^{\star}$ & $16(13,19)$ & $17(15,20)$ & $17(13,21)$ \\
\hline \multicolumn{4}{|l|}{ PUR welding: } \\
\hline Start, calendar year ${ }^{\star}$ & $1980(1970,1987)$ & $1974(1968,1984)$ & - \\
\hline Cumulative working days ${ }^{\star}$ & $755(240,2710)$ & $340(40,2820)$ & - \\
\hline Working days past month ${ }^{\star}$ & $7.8(0,20)$ & - & - \\
\hline End of exposure* & - & $1988(1979,1991)$ & - \\
\hline \multicolumn{4}{|l|}{ Ordinary welding: } \\
\hline Start, calendar year* & $1975(1954,1986)$ & $1959(1948,1974)$ & - \\
\hline Cumulative working days ${ }^{\star}$ & $1528(0,5390)$ & $2690(930,5280)$ & - \\
\hline Presently exposed (n (\%)) & $32(73)$ & $83(64)$ & - \\
\hline Working days past month ${ }^{\star}$ & $7.5(0,20)$ & $5(0,20)$ & - \\
\hline
\end{tabular}

$\star$ Median (10th, 90th percentile).

ing 65 men we obtained a response to the enquiry from 41.

Exposure data were obtained from a questionnaire and at a personal interview (see below for details). Fifty men ( $31 \%$ of the participants) had been engaged in PUR pipelaying during the three months immediately preceding the examination day. These men formed a subset of presently active PUR pipelayers. The remaining 113 men are here called formerly active PUR pipelayers. Of these, 89 men were still working as pipelayers, whereas 24 men had left the profession six months or more before the investigation.

The presently active PUR pipelayers were further subdivided into one group with recent high exposure - that is, $>10$ working days of PUR welding during the month preceding the examination ( $\mathrm{n}=19$; median 20 work days) and one group with recent low exposure - that is, $\leqslant 10$ work days, $(n=31$; median 0 work days).

Table 1 gives descriptive data on exposure for all participating pipelayers. The presently active PUR pipelayers had a higher estimate for lifetime cumulative days of PUR welding than the formerly active men, but a lower estimate for cumulative days of ordinary welding. Within the group of presently active PUR pipelayers a similar difference was noted; those with recent high exposure had as a median 1640 (10th, 90th percentile 390,3920 ) lifetime PUR welding days, whereas the group with recent low exposure had $500(130,1740)$ such days. The corresponding estimated cumulative working days with ordinary welding were 190 $(0,2100)$ and $2400(480,5900)$ respectively Even though the men with recent high exposure were younger than those with recent low exposure $(33(22,53) v 44(25,53)$ years $)$, the median duration of PUR exposure was similar ( 11 years in both groups).

The examination took place at the end of 1991 and the beginning of 1992 . Shortly afterwards, many pipelayers terminated their work because of a crisis in the building sector. Hence, in 1993 the questionnaire was once again sent to the group of 50 presently active PUR pipelayers, and was returned by 44 of them. Seventeen were still active in PUR work, whereas 27 had finished work. These groups did not differ with respect to age. Data obtained in 1991-2 indicated that these groups had similar tobacco consumption, whereas the men still active in 1993 had higher cumulative exposure to PUR (825 (280, 4000) days) compared with those who had finished working (645 (192, 2622) days).

Generally, there were two main categories of PUR pipelayers. One category consisted of pipelayers almost exclusively engaged in the construction of district heating pipe systems. Often they were employed in small companies, specialised in this type of job. At the time of investigation about $40 \%$ of the presently active PUR pipelayers were of this type.

For the rest, the main type of work task was mixed pipelaying and plumbing, including the welding of ordinary non-insulated pipes. Now and then they welded PUR insulated pipes, when connecting the internal pipe system of a building with the district heating pipe system. In the 1970s, when district heating pipe systems were first installed in many communities, PUR pipelaying occurred more often than nowadays for several of these men.

Earlier work practices often included the burning off of the foam insulation by a gas torch before welding; such practices were reported by one third of the pipelayers in our study. Later on, careful manual removal of at least $25 \mathrm{~cm}$ of the foam before welding was recommended. Either gas or manual metal arc welding was used. The use of personal protection devices had been very limited; only $7 \%$ of the men reported that they often or always used a half mask respirator. Foaming at the junction sites after welding was usually performed by specialised workers not included in the present study, but $15 \%$ of the pipelayers reported that they often or always used to foam MDI-based PUR.

\section{CONTROLS}

During the same time period as the pipelayers were examined, we investigated the metacholine reactivity in a working population, not presently exposed to airway irritants. This population included both blue and white collar workers from a pharmaceutical company, companies producing medical equipment and printed matters, shop assistants, postmen, paramedics, and radiology technicians. Volunteers were recruited, the only exclusion criteria being "almost daily use of asthma medications or use of peroral cortisone medication for any other reason". Only lifelong non-smokers and present smokers were eligible. For the present study, we selected all men in the same age range as the pipelayers as controls, in total 65 men (table 1).

\section{QUESTIONNAIRES}

A detailed questionnaire was sent by mail to all participants (pipelayers and controls) about two weeks before the examination. Major respiratory symptoms (dyspnoea, wheezing, and cough; translated from the British Medical Research Council questionnaire ${ }^{9}$ ), own and 
family allergy, previous pulmonary or cardiac disease, and questions directed at symptoms indicative of non-specific bronchial hyperreactivity were recorded. A lifetime employment history was reported, as were exposures to specified dusts, chemicals, and contact with animals during work or leisure time. The questionnaire also consisted of additional questions on the prevalence and frequency of specified symptoms from the eyes, nose, throat, and chest. Symptoms during the past year and earlier in adult life (for the pipelayers explicitly stated as the period before PUR pipelaying started) were recorded separately.

A detailed smoking history was also obtained from the questionnaire. The smoking habits among the pipelayers cannot be directly compared with those among the controls, as ex-smokers were excluded by definition from the second group. However, the daily amount of tobacco smoked was similar among active smokers, regardless of exposure state (table 1).

\section{INTERVIEW}

In a standardised interview at the examination a trained occupational health nurse (KKD) obtained information from the pipelayers about their detailed work history and their work habits during pipelaying and welding operations. Thus, the lifetime cumulative number of working days and the number of working days with welding of PUR pipes and welding of other pipes (also including other welding experiences) could be calculated. The corresponding figures were obtained for the preceding three months and one month. Days with part time work with a certain task were converted to eight hour working days. Supplementing questions on specific symptoms from the eyes, nose, throat, and chest, and their relation to PUR welding were also asked, without regard to noted respiratory complaints in the self administered questionnaire.

SKIN PRICK TEST AND ASSAYS OF SPECIFIC

IMMUNOGLOBULINS E AND G

For pipelayers and controls a skin prick test with 13 standard extracts (ALK, Copenhagen), a positive (histamine, $3 \mathrm{Hep}$ ), and a negative control was performed. For the pipelayers additional skin prick tests with isocyanate conjugates with human serum albumin were added. $^{6}$ The specific isocyanates used were 4,4'-MDI (which is normally the main component in technical grade MDI), toluene diisocyanate (commercial grade; 2,4- and 2,6-isomers), phenylisocyanate, p-toluene monoisocyanate, and 1,6-hexamethylene diisocyanate. The wheal size and shape were recorded on a transparent tape after 15 minutes. A wheal that was at least half the size of the histamine wheal was scored as positive. Atopy was defined as a positive skin reaction to at least one of the standard extracts. There was no difference between the PUR pipelayers and the controls in the prevalence of atopy (22\% v 25\%).

Radio allergosorbent test (RAST) and enzyme linked immunosorbent assay (ELISA) tests for specific IgE and IgG antibodies respectively, against MDI conjugates with human serum albumin were also performed, including serum samples from 49 of the controls. $^{6}$ For IgE-MDI, specific binding $>0.3 \%$ was considered positive (highest control $0.1 \%$ ). For IgG-MDI, values exceeding the highest value in the controls (IgG-MDI 0.14 A) were regarded as positive.

\section{LUNG FUNCTION TEST}

Vital capacity (VC) and forced expiratory volume during one second $\left(\mathrm{FEV}_{1}\right)$ were determined before and 15 minutes after administration of a B-agonist (two inhalations of Bricanyl Turbohaler). The lung function tests were performed as recommended by the American Thoracic Society, ${ }^{10}$ using a Vitalograph. The same test procedure was used for pipelayers and controls. The exposure state was not blinded for the technician performing the test. The VC and FEV , were expressed as \% predicted, using an external reference equation. ${ }^{11}$ Metacholine reactivity was also investigated (results not reported here).

\section{STATISTICS}

The prevalence odds ratio (POR), adjusted for potential confounders was used for measuring the effect of exposure to PUR. ${ }^{12}$ The focus of interest was on the one year prevalence of different types of symptoms. Effect estimations were performed by logistic regressions. Age ( $\leqslant 45,>45$ years), atopy (yes, no), and smoking (lifelong non-smoker, ex-smoker, smoker) were considered. Several categorical exposure variables were explored. The categorisations were as follows: exposure state (PUR pipelayer, control), present exposure state (presently active, formerly active), cumulative days for PUR welding $(\leqslant 500,>500)$, cumulative days for ordinary welding $(\leqslant 2500,>2500)$, days with PUR welding last month, $\leqslant 10,>10$ ), years since end of PUR welding $(0.25-5, \geqslant 5)$. For the lung function variables linear regressions, adjusted similarly for potential confounders, were performed. Here, age was treated as a continuous variable. Ex-smokers were omitted from the analyses when pipelayers and controls were compared, whereas they were included in the internal comparisons.

For statistical testing we used Fisher's exact test for binary variables, and the MannWhitney $U$ test for continuous variables. All tests were two tailed. The different statistical methods were performed as generally described. ${ }^{13}$

\section{Results}

SYMPTOMS

The prevalence of episodes (more than once a month) of irritative eye symptoms, congestion of the nose (blocked nose), and soreness or dryness in the throat (throat irritation) during the year preceding the examination was much higher among the PUR pipelayers than among the controls (table 2). This was especially pronounced among the non-smokers. Also, the pipelayers reported a higher prevalence of symptoms from the lower airways, such as pro- 
Table 2 Symptoms during the year preceding the examination among PUR pipelayers and controls (questionnaire data)

\begin{tabular}{|c|c|c|c|c|c|c|c|c|c|c|c|c|c|}
\hline & \multicolumn{6}{|c|}{ PUR pipelayers } & \multicolumn{4}{|c|}{ Controls } & \multicolumn{3}{|c|}{ Pipelayers $v$ controls } \\
\hline & \multicolumn{2}{|c|}{$\begin{array}{l}\text { Non-smokers } \\
(n=43)\end{array}$} & \multicolumn{2}{|c|}{$\begin{array}{l}\text { Ex-smokers } \\
(n=78)\end{array}$} & \multicolumn{2}{|c|}{$\begin{array}{l}\text { Smokers } \\
(n=42)\end{array}$} & \multicolumn{2}{|c|}{$\begin{array}{l}\text { Non-smokers } \\
(n=34)\end{array}$} & \multicolumn{2}{|c|}{$\begin{array}{l}\text { Smokers } \\
(n=31)\end{array}$} & \multirow{2}{*}{$\begin{array}{l}\text { Non-smokers } P R \\
(95 \% \text { CI })\end{array}$} & \multirow[b]{2}{*}{$\begin{array}{l}\text { Smokers } P R \\
(95 \% C I)\end{array}$} & \multirow[b]{2}{*}{$\begin{array}{l}\text { Adjusted POR } \\
(95 \% \mathrm{CI})\end{array}$} \\
\hline & $n$ & Prevalence & $n$ & Prevalence & $n$ & Prevalence & $n$ & Prevalence & $n$ & Prevalence & & & \\
\hline Eye irritation* & 11 & 0.26 & 16 & 0.21 & 11 & 0.26 & 3 & 0.09 & 3 & 0.10 & $2.9(0.9$ to 9.6$)$ & $2.7(0.8$ to 8.9$)$ & $3.6(1.3$ to 9.5$)$ \\
\hline Blocked nose* & 12 & 0.28 & 14 & 0.18 & 9 & 0.21 & 2 & 0.06 & 4 & 0.13 & $4.7(1.1$ to 19$)$ & $1.7(0.6$ to 4.9$)$ & $3.7(1.4$ to 10$)$ \\
\hline Throat irritation ${ }^{\star}$ & 10 & 0.23 & 6 & 0.08 & 4 & 0.10 & 1 & 0.03 & 1 & 0.03 & $7.9(1.0$ to 59$)$ & $3.0(0.4$ to 25$)$ & $6.8(1.4$ to 32$)$ \\
\hline Dry cough $\star$ & 6 & 0.14 & 10 & 0.13 & 6 & 0.14 & 2 & 0.06 & 7 & 0.23 & $2.4(0.5$ to 11$)$ & $0.6(0.2$ to 1.7$)$ & $1.0(0.4$ to 2.7$)$ \\
\hline Productive cough $†$ & 3 & 0.07 & 8 & 0.10 & 6 & 0.14 & 1 & 0.03 & 1 & 0.03 & $2.4(0.3$ to 22$)$ & $4.4(0.6$ to 35$)$ & $3.8(0.8$ to 1.9$)$ \\
\hline $\begin{array}{l}\text { Attacks of wheezing } \\
\text { or dyspnoea }\end{array}$ & 6 & 0.14 & 23 & 0.29 & 11 & 0.26 & 1 & 0.03 & 3 & 0.10 & $4.7(0.6$ to 38$)$ & $2.7(0.8$ to 8.9$)$ & $3.8(0.2$ to 12$)$ \\
\hline
\end{tabular}

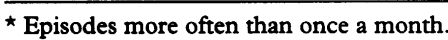

† On most days for more than three months.

$¥$ Ever such episodes.

$\mathrm{PR}=$ prevalence ratio; $\mathrm{POR}=$ prevalence odds ratio; from a logistic regression, controlling for age, atopy, and smoking habits (ex-smokers excluded).

Table 3 Prevalence of symptoms during the year preceding the examination among presently active and former PUR pipelayers (questionnaire data)

\begin{tabular}{|c|c|c|c|c|c|}
\hline & \multirow{2}{*}{\multicolumn{2}{|c|}{ Presently active }} & \multicolumn{2}{|c|}{ Formerly active } & \multirow{3}{*}{$\begin{array}{l}\text { Presently active } \\
\text { formerly active } \\
\text { (adjusted) POR } \\
(95 \% \text { CI) }\end{array}$} \\
\hline & & & \multicolumn{2}{|c|}{ Time since end of exposure } & \\
\hline & $\begin{array}{l}\text { Recent high } \\
\text { exposure }(n=19)\end{array}$ & $\begin{array}{l}\text { Recent low } \\
\text { exposure }(n=31)\end{array}$ & $\begin{array}{l}0.25-5 y \\
(n=64)\end{array}$ & $\begin{array}{l}>5 y \\
(n=49)\end{array}$ & \\
\hline Eye irritation* & 0.37 & 0.32 & 0.20 & 0.16 & $2.9(1.2$ to 6.9$)$ \\
\hline Blocked nose & 0.37 & 0.26 & 0.16 & 0.20 & $1.6(0.7$ to 3.9$)$ \\
\hline Throat irritation $\star$ & 0.32 & 0.13 & 0.11 & 0.06 & $2.0(0.7$ to 6.1$)$ \\
\hline Dry cough $\star$ & 0.16 & 0.16 & 0.16 & 0.08 & $1.1(0.4$ to 3.2$)$ \\
\hline Productive cought & 0.16 & 0.13 & 0.08 & 0.10 & $2.6(0.8$ to 8.4$)$ \\
\hline Attacks of wheezing or dyspnoeał & 0.32 & 0.19 & 0.25 & 0.24 & $1.3(0.5$ to 3.1$)$ \\
\hline
\end{tabular}

* Episodes more often than once a month.

t On most days for more than three months.

$\ddagger$ Ever such episodes.

POR = prevalence odds ratio, obtained from a logistic regression, controllin for age, atopy, smoking habits, and cumulative exposure (PUR and other welding).

ductive cough (on most days for more than three months a year), attacks of dyspnoea and wheezing (ever such episodes). Logistic regression models, including age, atopy, and smoking habits in addition to exposure status (pipelayer or control) confirmed the findings.

The presently active PUR pipelayers reported the highest prevalence of eye, nose, and

Table 4 Relation between symptoms and PUR pipe laying among 163 pipelayers (interview data)

\begin{tabular}{lllcl}
\hline & $\begin{array}{l}\text { No symptoms } \\
\text { at work }\end{array}$ & $\begin{array}{l}\text { Symptoms } \\
\text { appearing } \\
\text { immediately }\end{array}$ & $\begin{array}{l}\text { Symptoms } \\
\text { appearing in } \\
\text { the evening }\end{array}$ & $\begin{array}{l}\text { Better on } \\
\text { weekends }\end{array}$ \\
\hline Eye irritation & 114 & 41 & 8 & 42 \\
Blocked nose & 134 & 17 & 12 & 12 \\
Throat irritation & 130 & 28 & 5 & 28 \\
Dry cough & 144 & 17 & 2 & 17 \\
Attacks of wheezing or dyspnoea & 141 & 14 & 8 & 16 \\
\hline
\end{tabular}

* Or immediately and in the evening.

Table 5 Prevalence of symptoms during the preceding year in 1991-2 and in 1993 in relation to exposure state 1993 among 44 PUR pipelayers, who were all active in 1991-2 (questionnaire data)

\begin{tabular}{|c|c|c|c|c|}
\hline & \multicolumn{2}{|c|}{ Questionnaire 1991-2 } & \multicolumn{2}{|c|}{ Questionnaire 1993} \\
\hline & $\begin{array}{l}\text { Active } 1993 \\
(n=17)\end{array}$ & $\begin{array}{l}\text { Not active } 1993 \\
(n=27)\end{array}$ & $\begin{array}{l}\text { Active } 1993 \\
(n=17)\end{array}$ & $\begin{array}{l}\text { Not active } 1993 \\
(n=27)\end{array}$ \\
\hline Eye irritation* & 0.41 & 0.19 & 0.47 & 0.22 \\
\hline Blocked nose* & 0.35 & 0.15 & 0.35 & 0.22 \\
\hline Throat irritation * & 0.29 & 0.11 & 0.23 & 0.15 \\
\hline Dry cough ${ }^{\star}$ & 0.18 & 0.07 & 0.12 & 0.04 \\
\hline \multirow{3}{*}{$\begin{array}{l}\text { Productive cought } \\
\text { Attacks of wheezing or } \\
\text { dyspnoea } \ddagger\end{array}$} & 0.18 & 0.07 & 0.04 & 0.07 \\
\hline & & & & \\
\hline & 0.17 & 0.07 & 0.18 & 0.15 \\
\hline
\end{tabular}

* Episodes more often than once a month

† On most days for more than three months.

$\ddagger$ Ever such episodes. throat symptoms (table 3). Logistic regression models, including age, atopy, and smoking habits in addition to present exposure status confirmed the findings, which, however, reached significance only for eye irritation. The prevalences of these symptoms decreased with increasing time since the end of PUR pipelaying. In contrast, there was no difference in the prevalence of symptoms from the lower airways between presently or formerly active PUR pipelayers (table 3 ). In logistic regression models, the cumulative days of PUR welding and ordinary welding were explored, showing no significant associations with any type of symptoms. Nominally higher prevalences of symptoms were reported among presently active pipelayers with recent high, compared to those with recent low PUR exposure.

In the interview, most of the pipelayers with symptoms reported that these had started and occurred in relation to the PUR welding tasks (table 4). The non-atopic workers reported work related symptoms as often as the atopic workers; likewise there was no difference in the reports between workers with different smoking habits. The interval between start of PUR welding and the occurrence of any work related symptom was long, with a median $9(2,20)$ years. Atopic workers did not report a shorter symptom free interval than did non-atopic workers.

Table 5 shows the results from the 1993 questionnaire. The symptom prevalences were stable over time. 
SKIN PRICK TEST AND ASSAYS OF SPECIFIC IMMUNOGLOBULINS E AND G

None of the pipelayers showed positive skin prick reactions against the specific isocyanate antigens used or positive IgE-MDI. Two pipelayers showed low IgG-MDI values $(0.18$ and $0.30 \mathrm{~A}$ ).

\section{LUNG FUNCTION}

Table 6 shows $\mathrm{VC}$ and $\mathrm{FEV}_{1}$ (expressed as $\%$ predicted) among all pipelayers and controls. In multiple linear regression models (using the observed value, adjusted for smoking habits, age, and height) a small effect of exposure was found for $\mathrm{FEV}_{1} \quad(\beta=-0.1$ $1(P=0.10)$ ) but not for VC (table 6). As expected, smoking effects were evident for both $\mathrm{FEV}_{1}$ and VC.

Similarly, when presently active pipelayers were compared with controls, the corresponding effect for $\mathrm{FEV}_{1}$ was $B=-0.21(\mathrm{P}=0.09)$. The effect was most pronounced among presently active pipelayers with recent high exposure to PUR, having an estimated reduction of $\mathrm{FEV}_{1}$ of $-0.31(\mathrm{P}=0.04)$, compared to controls. The reduction among those with recent low exposure to PUR was less, $(-0.11(P>0.2))$. In the models, the estimated reduction among smokers was about $-0.41(\mathrm{P}<0.001)$. No confounding effect of ordinary welding could be demonstrated. For VC no differences between pipelayers and controls were evident.

In internal comparisons among the pipelayers, including also ex-smokers, there were no significant differences in $\mathrm{FEV}_{1}$ and $\mathrm{VC}$ between presently and formerly active PUR pipelayers, adjusting for age, height, and smoking habits. We explored the effects of several other exposure indices (cumulative days of PUR welding, cumulative days of ordinary welding, time since end of PUR and ordinary welding) in further models, but no significant exposure-response relations were disclosed for any of them. Likewise, a similar internal comparison between men with recent high and recent low PUR exposure did not show significant differences in $\mathrm{FEV}_{1}$.

\section{NON-PARTICIPANTS}

According to information from the initial short enquiry, or from the employer's lists, the duration of PUR pipelaying was estimated to have been 1-12 months for 17 (26\%) of the 65 non-

Table 6 Lung function in pipelayers and controls

\begin{tabular}{|c|c|c|}
\hline & $V C$ & $F E V_{l}$ \\
\hline \multicolumn{3}{|c|}{ Lung function, expressed as \% predicted ${ }^{\star}$ : } \\
\hline Non-smokerst & $90(78,101)$ & $100(87,115)$ \\
\hline Ex-smokerst & $85(71,102)$ & $97(76,115)$ \\
\hline Smokerst & $88(74,103)$ & $91(74,109)$ \\
\hline \multicolumn{3}{|l|}{ Controls: } \\
\hline Non-smokers $†$ & $95(85,104)$ & $104(91,114)$ \\
\hline Smokerst & $88(75,104)$ & $95(79,107)$ \\
\hline \multicolumn{3}{|c|}{ Linear regression modelsł: } \\
\hline Exposure state & $-(>0.10)$ & $-0.12(0.10)$ \\
\hline Smokings & $-0.19(0.02)$ & $-0.38(<0.01)$ \\
\hline Atopy5 & $-(>0.10)$ & $-(>0.10)$ \\
\hline
\end{tabular}

* Reference equation from Berglund $e t a l^{11}$

† Median (10th, 90th percentile).

‡ Ex-smokers excluded; adjusted also for age, and height.

$\beta \beta$ (in litres) ( $P$ value). participants, and $>1$ year for five ( $8 \%$ ) of them. For the 163 participants the corresponding figures, as obtained from these sources, were $34 \%$ and $45 \%$. Among the 41 non-participants who responded to the short initial enquiry three men $(7 \%)$ reported work related symptoms from the eyes or nose, and five men (12\%) reported work related airway symptoms. In contrast, among the participants the corresponding figures were $32 \%$ and $43 \%$ respectively.

\section{Discussion}

We have found that PUR pipelayers, compared with unexposed controls, experienced an increased prevalence of current irritative symptoms from the eyes, nose, and throat. They also reported that such symptoms occurred in direct relation to PUR welding. The prevalence of irritative symptoms was highest among the presently active PUR pipelayers, and decreased with increasing time since last PUR exposure. The same pattern emerged for productive cough. A direct irritating effect of thermal degradation products of MDI based PUR is the most likely explanation to these findings.

The presently active pipelayers with recent high PUR exposure had a reduced $\mathrm{FEV}_{1}$ compared with controls. A reduction of $F E V_{1}$ also occurred in the total PUR pipelayer population, but it was weaker. Attacks of wheezing or dyspnoea, symptoms indicating airway obstruction, were nominally more prevalent among pipelayers than among controls, but not more prevalent among presently $v$ formerly active pipelayers. When assessing the prevalence of respiratory symptoms and signs in a cross sectional study population, such as the subset of presently active pipelayers, a selection bias may be introduced, because workers with respiratory symptoms and signs may have left the PUR work task beforehand. This will lead to an underestimation of the true risk. This bias may be present in the direct comparisons between presently and formerly active pipelayers, but is reduced in the overall analyses in which formerly active PUR pipelayers are included.

An aggravation of respiratory symptoms from concomitant exposure to respiratory irritants at work and smoking may increase the tendency for smokers to quit their habit. We have reason to think that such interactions have affected our results, as we found four exsmokers who had a clinical diagnosis of asthma during their work as PUR pipelayers, and one ex-smoker who had his asthma diagnosis while he was working as a pipelayer but before PUR welding started. All but one of them reported aggravated symptoms during PUR welding, whereas the remaining subject stated that ordinary welding was equally symptom provoking. Thus, this may have resulted in an underestimation of the true risk for symptoms and decreased lung function in the direct comparisons between pipelayers and controls, as all ex-smoking pipelayers were excluded. Four of the five ex-smoking asthmatic workers also belonged to the formerly active group. This 
could partly explain why we found no clearcut findings indicating a persisting adverse effect of exposure to the lower airways.

The basis for recruitment to the study was company reports of employees. We had no possibility of investigating the completeness of these reports. However, for men still active as pipelayers or having related assignments, we think that the losses should have been small, as we recruited from all companies specialising in PUR pipelaying, and all other major pipelaying companies in the area. For pipelayers entirely leaving their occupation there might be some losses, which we cannot estimate. For identified subjects who were non-participants, we have some information, indicating less exposure and less symptoms, than among participants.

Another bias which may be operating is a selection of healthy persons to the occupation. It was found that welders under the age of 30 had a higher $\mathrm{FEV}_{1}$ than a group of controls. ${ }^{14} \mathrm{In}$ this study we have no indications of a major selection bias of this type. We did not find a difference in the prevalence of atopy, or a difference in the proportion of men with symptoms earlier in adult life or before PUR work started when all PUR pipelayers and controls were compared. We also found that VC and $\mathrm{FEV}_{1}$ among controls and formerly active PUR pipelayers under the age of 35 were very close (data not given). In contrast, young presently active PUR pipelayers showed a reduction of $\mathrm{FEV}_{1}$ compared with controls. This is again comparable with an exposure effect.

Can we then attribute the excess of respiratory symptoms and decreased lung function in the pipelayers to their exposure to thermal degradation products from isocyanates? The investigated pipelayers were exposed to a complex mixture of compounds, several of which are well known respiratory irritants or sensitisers. High peak exposures to isocyanates and amines occur at thermal decomposition of technical quality MDI-based PUR, which in itself has a complex isomeric composition, and more complex and new isocyanate compounds are formed. ${ }^{7}$ Aldehydes and other compounds are formed from the degraded polyol ingredient. Further, the gas or metal arc welding of the mild steel pipes, which are sometimes coated by a protective oil, yields various gaseous and particulate air contaminants. ${ }^{15} \mathrm{~A}$ recent overview of the literature on respiratory effects of welding was presented by Sjögren and Ulfvarson. ${ }^{15}$ The prevalence of chronic (simple) bronchitis has repeatedly been increased among welders. Data are, however, conflicting as to whether non-smoking welders also have an increased prevalence of chronic bronchitis. Impaired spirometric values have been found mainly for $\mathrm{FEV}_{1}$. In some studies the effect was seen only in smokers, in others non-smokers have also been affected. ${ }^{16}$ Although the interest in respiratory effects of welding fumes have focused on the bronchi, it is evident that the upper airways can also be affected. An increased prevalence of symptoms from the upper respiratory tract has been described, with chronic rhinitis as the most often reported symptom among shipyard welders. ${ }^{17}$

The outlined complexity of exposure indeed merits caution in the internal comparisons. We estimated the cumulative work days of PUR and ordinary welding, based on the retrospective self reports. These estimates are by nature subject to uncertainties. Time since last exposure to PUR is probably more accurately determined. We also presume that recent working days can be accurately reported. Other factors which could not be taken into account, not least individual work habits, may greatly influence the exposure to the thermal degradation products of MDI and the welding fumes. We have previously shown, in this study base of pipelayers, that the number of welded PUR pipes during the preceding three months (which closely parallels duration of such work) correlates positively with 4,4-methylenedianiline in plasma, a biomarker for exposure. ${ }^{8}$ The present study group consists of pipelayers with varying intensity and duration of PUR pipelaying and ordinary welding, a prerequisite for separation of the effects, but our exposure indices are crude. This may partly explain the difficulties in showing exposure-response relations in the internal comparisons. However, in the group of presently active pipelayers it was obvious that the group with recent high exposure to PUR had a greater decrement of $F E V_{1}$ than the controls, whereas the group with recent low exposure had not. This finding cannot be explained by concomitant ordinary welding, as these men actually performed less ordinary welding during the preceding month, and also had fewer cumulative days of ordinary welding, than the pipelayers with ongoing recent low PUR exposure.

We did not find any evidence of IgE antibodies in any of the pipelayers, and only two of them had antibodies of the IgG type. This is not remarkable, as the prevalence of specific antibodies may be low in cohorts of workers exposed to MDI. ${ }^{6}{ }^{18}$

The pipelayers were exposed to a complex mixture of inhaled irritants. We have not tried to evaluate whether any of the workers included in the present study have "isocyanate asthma" in a strict sense. "Variable airflow limitation causally related to exposure to non-irritant levels of isocyanates", 19 cannot ultimately be diagnosed without specific inhalation challenges. Neither questions on the occurrence and timing of obstructive symptoms in relation to work, ${ }^{20}$ nor specific serum antibodies, ${ }^{21}$ are sensitive and specific enough for a strict diagnosis.

In summary, as in other groups of workers, ${ }^{6}$ exposure to thermal degradation products from MDI based polyurethane among these PUR pipelayers was related to adverse effects. The symptoms and decrease in lung function cannot be explained by ordinary welding exposure only. We found that four out of five men who had obtained a clinical diagnosis of asthma during PUR pipelaying or ordinary pipelaying work were atopic. However, we did not find substantial differences in prevalence of symptom, reported work related symptoms, or 
lung function between pipelayers with and without atopy. Thus, as has been pointed out before, ${ }^{22}$ pre-employment removal of atopic workers out of the workforce cannot prevent adverse exposure effects. The only possibility is to minimise the exposure to thermal degradation products by using optimal working procedures.

We acknowledge the cooperation of all the pipelayers and the companies. We thank Assistant Professor Hans Welinder for assistance with the immunological analyses. Grants were obtained from the Swedish Council for Work Life Research and the Faculty of Medicine, Lund University. The study was approved of by the Ethics Committee, Faculty of Medicine, Lund University.

1 Meredith SK, Taylor VM, McDonald JC. Occupational respiratory disease in the United Kingdom 1989: a report to the British Thoracic Society and the Society of Occupational Medicine by the SWORD project group. Br $f$ Ind Med 1991;48:292-8.

2 Longley EO. Methane diisocyanate: a respiratory hazard? Arch Environ Health 1964;8:898.

3 Tanser AR, Bourke MP, Blandford AG. Isocyanate asthma: respiratory symptoms caused by diphenyl-methane diisocyanate. Thorax 1973;28:596-600.

4 Banks DE, Balaan MR. The respiratory effects of isocyanates. In: Rom W, ed. Environmental and occupational medicine. 2nd ed. Boston: Little Brown, 1992:967-86.

5 Vandenplas O, Malo J-L, Saetta M, Mapp CE, Fabbri LM Occupational asthma and extrinsic alveolitis due to Occupational asthma and extrinsic alveolitis due to isocyanates: curre

6 Skarping G, Dalene M, Svensson BG, Littorin M, Åkesson $B$, Welinder H, Skerfving S. Biomarkers of exposure, antibodies, and respiratory symptoms in workers heating polyurethane glue. Occup Environ Med 1996;53:180-7.

7 Tinnerberg H, Spanne M, Dalene M, Skarping G. Determination of complex mixtures of airborne isocyanates and amines. Part 3. Methylenediphenyl diisocyanate, methylen(a) (henyldiamine and structural analogues after therm
of polyurethane. Analyst 1997;122:275-8.
8 Dalene M, Jakobsson K, Rannug A, Skarping G, Hagmar L. MDA in plasma as a biomarker of exposure to pyrolysed MDI-based polyurethane: correlations with estimated cumulative dose and genotype for $\mathrm{N}$-acetylation. Int Arch Occup Environ Med 1996;68:165-9.

9 Medical Research Council. Definition and classification of chronic bronchitis for clinical and epidemiological purposes. Lancet 1965;i:775-9.

10 American Thoracic Society. Standardization of spirometry: 1987 update. Am Rev Respir Dis 1987;136:1285-98.

11 Berglund E, Birath G, Bjure J, Grimby G, Kjellmer I, Sandqvist L, Söderholm B. Spirometric studies in norma subjects. I. Forced expirograms in subjects between 7 and 70 years of age. Acta Med Scand 1963;173:185-98.

12 Strömberg U. Prevalence odds ration $v$ prevalence ratio some further comments [letter]. Occup Environ Med 1994 52:143.

13 Checkoway H, Pearce NE, Crawford-Brown DJ. Research methods in occupational epidemiology. Oxford: Oxford methods in occupational
University Press, 1989.

14 Cotes JE, Feinmann EL, Male VJ, Rennie FS, Wickham CAC. Respiratory symptoms and impairment in shipyard welders and caulker/burners. Br F Ind Med 1989;46:292301.

15 Sjögren B, Ulfvarson U. Welding gases and fumes. In: Beije B, Lundberg P, eds. Criteria documents from the Nordic Expert Group 1990. Arbete och Hälsa 1991;2:281-315.

16 Kilburn KH, Warshav RH. Pulmonary functional impairment from years of arc welding. Am $\mathcal{F}$ Med 1989;87:62-9.

17 Kalliomäki P-L, Kalliomäki K, Korhonen O, Nordman H, Rahkonen E, Vaaranen V. Respiratory status of stainless steel and mild steel welders. Scand $\mathcal{f}$ Work Environ Health 1982;8(suppl 1):117-21.

18 Liss GM, Bernstein DI, Moller DR, Gallagher JS, Stephenson RL, Bernstein IL. Pulmonary and immunologic evaluation of foundry workers exposed to methylene diphenyldiison of foudr.

19 Newman Taylor AJ. Occupational asthma. Thorax 1980;35: 241-5.

20 Malo J-L, Ghezzo H, L'Archeveque J, Lagier F, Perrin B, Cartier A. Is the clinical history a satisfactory means of diagnosing occupational asthma? Am Rev Respir Dis 1991 143:528-32.

21 Baur X, Marek W, Ammon J, Czuppon AB, Marczynski B Raulf-Heimsoth M, et al. Respiratory and other hazards of isocyanates. Int Arch Occup Health 1994;66:141-52.

22 Hendrick DJ. Management of occupational asthma. Eur Respir $\mathcal{F}$ 1994;7:961-8.

\section{Correspondence and editorials}

Occupational and Environmental Medicine welcomes correspondence relating to any of the material appearing in the journal. Results from preliminary or small scale studies may also be published in the correspondence column if this seems appropriate. Letters should be not more than $\mathbf{5 0 0}$ words in length and contain a minimum of references. Tables and figures should be kept to an absolute minimum. Letters are accepted on the understanding that they be subject to editorial revision and shortening.

The journal also publishes editorials which are normally specially commissioned. The Editor welcomes suggestions regarding suitable topics; those wishing to submit an editorial, however, should do so only after discussion with the Editor. 